\title{
Ultrasound Image Segmentation With Shape Priors: Application to Automatic Cattle Rib-Eye Area Estimation
}

\author{
Pablo Arias, Alejandro Pini, Gonzalo Sanguinetti, Pablo Sprechmann, Pablo Cancela, Alicia Fernández, \\ Alvaro Gómez, and Gregory Randall
}

\begin{abstract}
Automatic ultrasound (US) image segmentation is a difficult task due to the quantity of noise present in the images and the lack of information in several zones produced by the acquisition conditions. In this paper, we propose a method that combines shape priors and image information to achieve this task. In particular, we introduce knowledge about the rib-eye shape using a set of images manually segmented by experts. A method is proposed for the automatic segmentation of new samples in which a closed curve is fitted taking into account both the US image information and the geodesic distance between the evolving curve and the estimated mean rib-eye shape in a shape space. This method can be used to solve similar problems that arise when dealing with US images in other fields. The method was successfully tested over a database composed of 610 US images, for which we have the manual segmentations of two experts.
\end{abstract} tion.

Index Terms-Rib-eye, shape priors, ultrasound (US) segmenta-

\section{INTRODUCTION}

A UTOMATIC segmentation of ultrasound (US) images is an important problem with particular difficulties. The US images are very noisy and artifacts (e.g., bad defined boundaries, speckle noise) can appear due to errors in the acquisition process. Many times, it is difficult, even for a human interpreter, to decide the limits of a given region in these type of images. At the same time, the nonintrusive US capability is very useful in applications ranging from medical imaging to animal quality assessment.

In this paper, we propose an automatic algorithm for US images segmentation in the context of quality meat assessment. More precisely, we propose a shape prior based segmentation algorithm to estimate the rib-eye area.

Manuscript received May 17, 2006; revised December 28, 2006. This work was supported in part by CSIC (Universidad de la República), in part by ECE and IMA (University of Minnesota), in part by the National Science Foundation, in part by the Office of Naval Research, and in part by DARPA. The associate editor coordinating the review of this manuscript and approving it for publication was Dr. Gaudenz Danuser.

The authors are with the Instituto de Ingeniería Eléctrica, Universidad de la República, Montevideo 11300, Uruguay (e-mail: parias@fing.edu.uy; alejandro.pini@gmail.com; gsangui@fing.edu.uy; psprech@fing.edu.uy; pcancela@fing.edu.uy; alicia@fing.edu.uy; agomez@fing.edu.uy; randall@ fing.edu.uy).

Color versions of one or more of the figures in this paper are available online at http://ieeexplore.ieee.org.

Digital Object Identifier 10.1109/TIP.2007.896604
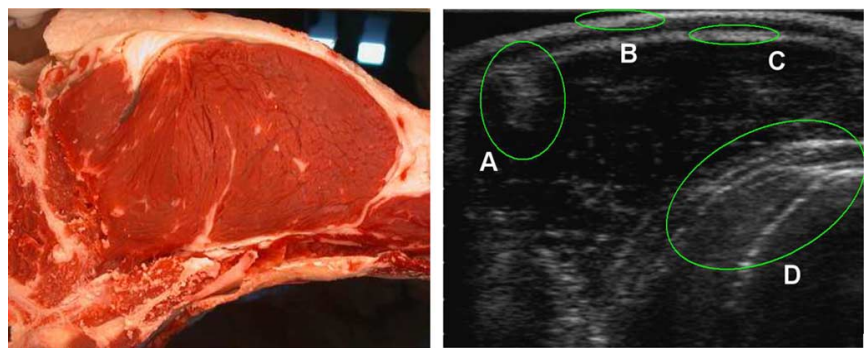

Fig. 1. Left: Image of a carcass cross section in which we can see the rib-eye. Right: Notable regions in a typical rib-eye US image. A: Hook (not always present); B: skin; C: subcutaneous fat; D: limits between longissimus dorsi muscle and the intercostal muscle.

The Yield Grade (YG) is a commonly used numerical representation of the expected percentage of closely trimmed, boneless retail cuts of the animal's round, loin, rib, and chuck [16]. The YG can be estimated through measurements in the still alive animal. Among these measurements are the rib-eye area, the subcutaneous fat and the intramuscular fat. Currently, these measurements are made through US images taken from the animal and analysed by experts [21]. There exists commercial software (SW) [19], [22] for the semiautomatic estimation of these measurements, but needs an expert to draw the rib-eye contour on the US image. In [25], the authors show results of an algorithm for the automatic estimation of the rib-eye area using US images, but they do not explain the proposed method. In [2], Cancela et al. proposed a method to solve this problem, that inspired our approach. The shape prior used in [2] was an $a d$ hoc construction and, although the experimental database was more limited, the results were encouraging. We have compared that algorithm with the one proposed in this paper, using a much more extensive database and attained significantly better results.

To the best of our knowledge, there are no works in the literature that present a robust, real time, automatic method for the rib-eye area estimation using US images. The aim of this work is to propose such an algorithm. Fig. 1 shows a typical US image as well as the corresponding carcass cross section and Fig. 8 shows two experts' manual segmentations of the rib-eye for some images.

The US images we work with are acquired in the field, often under tough conditions. The ambient humidity, the movement of the animal, and the experience of the operator, are among the factors that affect image quality. Generally speaking, in US images, density changes that are parallel to the US probe get 
the higher intensity values. Interface regions between tissues of similar characteristics or that are perpendicular to the probe are badly defined. Regions closer to the probe (the animal skin) have better definition. This phenomenon is illustrated in Fig. 1. The animal skin (region B) is well defined, as is the backfat marked C. Region D corresponds to the intercostal muscle, which separates the longissimus dorsi muscle (the cross-section of this muscle is the rib-eye) from the internal organs of the animal. Note that some limits of the rib-eye are well defined, while others are almost absent. The experts must be well trained in order to manually trace the US images and produce good results. In fact, the experts use the knowledge of the rib-eye shape to manually trace the US images.

In this paper, we propose a solution to this problem with an automatic algorithm inspired by the experts' approach. The idea is to evolve a curve in the image in such a way that its final position corresponds to the segmentation of the rib-eye. The curve evolution is driven by two terms, one uses features taken from the image, while the other includes a priori knowledge about the shape of the curve. The rib-eye shape is a closed simple curve, codified in the shape space framework proposed in [11]. In this framework, the shape is a point in a given manifold, in which the distance between shapes is the length of the geodesic path between the corresponding points in the manifold. The shape prior is the mean rib-eye shape, learned as the Karcher mean of a subset of the expert marked database, plus the principal variations from this mean shape. The shape step moves the current curve along the geodesic path towards the mean shape, while the image step deforms the curve locally in order to fit the well defined regions in the US image. The algorithm was tested on a set of 610 chosen US images, taken under very different conditions. The results are encouraging.

This paper is organized as follows. First, we briefly describe the general scheme of the algorithm. The following three sections explain the central parts of the proposed solution: the image correction step, the shape correction step, and the initial condition algorithm. Next, we discuss the algorithm's performance on a large set of real images. Finally, we conclude and propose some future work.

\section{General Description OF THE AlgORITHM}

The goal of the algorithm is to emulate the experts' methodology. From experience they have learned the typical shape of the rib-eye and its possible variations. To detect the rib-eye, they fit a known shape to the features present in the image, mostly the borders parallel to the probe. As seen in Fig. 1, this knowledge is essential to interpolate the curve in those regions with insufficient data. In order to incorporate the shape knowledge, we learn it using a set of expert traced curves.

Fig. 2 illustrates the proposed solution. We place an initial curve $C_{0}$, represented by a set of equidistant sampled points, the control points, in a given image. Given the difficulty of minimizing an energy functional combining information from different domains (image and shape space), we use an alternative method. The curve is iteratively deformed by two successive steps: image correction and shape correction. The process stops when a given condition is attained. The first step is related to the image and tries to move the curve towards the well defined

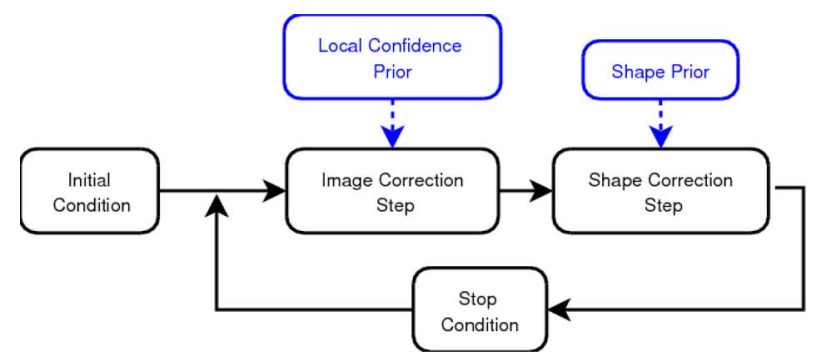

Fig. 2. Block diagram of the proposed automatic algorithm.

boundaries of the US image. The second step is related to the knowledge that we have about the rib-eye shape.

In recent years, several authors have proposed shape prior segmentation algorithms [7], [12], [18]. The general idea is always to include some a priori information about the shape in the segmentation algorithm in order to limit the space of possible solutions. Chen et al. [6] proposed a shape prior segmentation method using the curve representing the boundary of an object as the shape representation. The method introduces a shape prior in the Geodesic Active Contour framework proposed by Caselles et al. [3]. Rousson and Paragios [18] used a signed distance function as a shape representation. Several authors have used this shape representation in order to introduce the shape priors in their proposals [4], [7], [23]. Paragios [17] use a probabilistic level set distance map. Other shape distances have also been used, such as the Hausdorff distance in [5]. We use the framework proposed by Klassen et al. [11], to define a shape space $(\mathcal{S})$, where we can measure the similarity between shapes as well as model the statistics of the rib-eye's shape. This framework is well adapted to the case of simple closed curves, without landmarks, as is the case in our application. We can think of the shape of each closed curve as a point in an infinite-dimensional manifold $\mathcal{S}$. Distances between shapes are defined as the length of the geodesic paths between points in $\mathcal{S}$.

Two experts manually segmented the set of 610 US images taken from animals belonging to ten different farms. With a subset of these curves (the training set) we estimate a mean shape $\mu$ as the point in $\mathcal{S}$ which minimizes the sum of the squared geodesics distances to all the training set shapes (points in $\mathcal{S}$ ). The initial condition step roughly fits the mean shape to the data and produces an initial curve $C_{1}$. From there on, the current curve $C_{k}$ is deformed. The shape related step moves $C_{k}$ along a geodesic path in $\mathcal{S}$ towards $\mu$, a distance dependent on the probability of the shape of $C_{k}$ according to the learned model. The image related step then locally deforms $C_{k}$ in such a way that it passes through the well defined regions (muscle limit), where the discontinuities are more important. This produces a new curve $C_{k+1}$, which maps to a new point in $\mathcal{S}$, from where a new geodesic path to $\mu$ is generated and the whole process is repeated. The curve are expected to converge to a solution which is a good approximation to the experts' manual segmentation.

The stopping condition is based on the movement of the curve during the evolution process. The stop condition test is performed after both steps of each iteration are completed. The algorithm stops when the mean absolute difference between the 

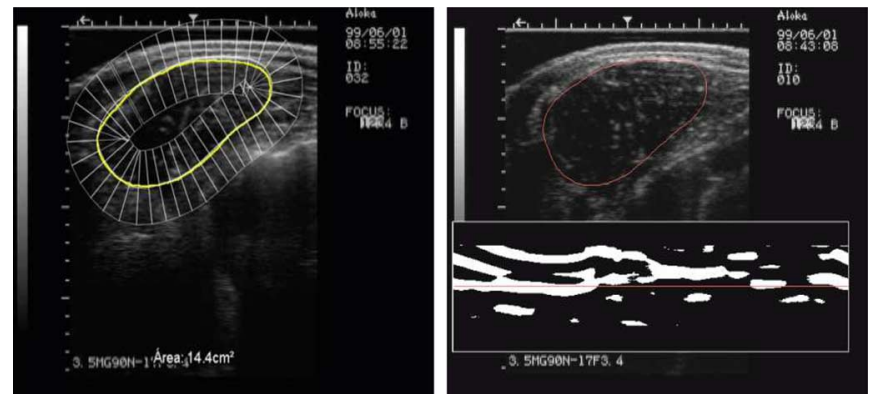

Fig. 3. Normal mapping of a curve $C$ is obtained by the unroll of a band along the curve $C$. Left: Band defined by a given curve. Right: Binarized normal mapping of that region.

control points of $C_{k}$ and $C_{k+1}$ is less than one pixel by control point or when it reaches a fixed number of iterations.

\section{IMAGE CORRECTION STEP}

\section{A. Normal Mapping}

It is important to permit strong local deformations of the curve in order to deform the curve toward the data of the images. To do so, we work on a band around the curve and allow for deformations in the normal direction to the curve. This allows us to account for the image information used by the experts when manually tracing the curves. It also restricts the use of the image information to those regions that matter in this process. To this end, we define a normal mapping (NM) as a coordinate transformation, dependent on a curve $C$. The idea is to unroll the band around the curve in such a way that the horizontal coordinate $u$ is the arc length of the curve and the vertical coordinate is the distance to the curve.

Let us denote by $(u, v)$ the coordinates in the NM space of a point $(x, y)$ in the band. The vertical coordinate $v$ is the distance from that point $(x, y)$ to the curve $C$. In other words, points in a vertical direction in NM, $u=u_{0}$, are in the normal direction to $C$ in the image. In this space, the curve $C$ is the horizontal axis. The coordinate transformation is depicted in Fig. 3. For details about the NM, please refer to [2].

\section{B. Learning the Confidence of the Image Information Along the Rib-Eye Limits}

Analyzing an US image, like the one in Fig. 1, we can see that the rib-eye limits are well defined near the skin, at the top of the image. The bottom region is less well defined and in the lateral regions there is almost no data.

To learn the regularity of the presence of borders we study the gray level information in the neighborhood along the expert traced curves. To achieve this aim, we took the expert marked curves on 180 US images from the training set, and applied the normal mapping to each one. Once in the NM space, we register the images and estimate their vertical gradients. We proceed next to pixel-wise average this set of images. Let us call $\overline{I_{N M}}$ the mean NM image, and $\overline{\nabla I_{N M}}$ the mean NM gradient image. The first row in Fig. 4 shows $\overline{I_{N M}}$. Regions with $u \in[450,600]$ are well contrasted. This means that for most of the images, the edges in these sections of the rib-eye's contour are well defined.

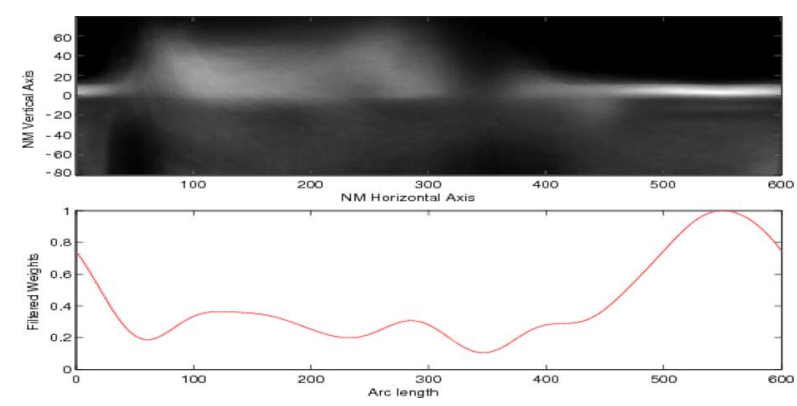

Fig. 4. Mean gray-level image $\overline{I_{N M}}$ of 180 images in the NM space. Weight function $F_{s c}$ along the curve.

In fact, these regions correspond to the upper side of the rib-eye. Using these two images, we produce a weight function

$$
F_{s c}(u)=K G_{\sigma} *\left(\overline{I_{N M}}(u, 0)+\overline{\nabla I_{N M}}(u, 0)\right)
$$

where $K$ is a normalization factor, $G_{\sigma}$ is a Gaussian filter, and $u$ is the arc length. The weight function is illustrated in the second row of Fig. 4. This precalculated function is used to modulate the confidence we have in the image information along the rib-eye contour.

\section{Image Correction}

In the image correction step of the algorithm, at iteration $k$, each control point of $C_{k}$ is moved along the normal direction by a magnitude that depends on the image in the neighborhood band. In this case, it depends on a weighted average of pixel properties along the normal to $C_{k}$. The weight takes in account the presence of bright regions roughly parallel to $C_{k}$.

A band of width $2 d$ around $C_{k}$ is mapped using the NM transformation. We apply to the NM transformed image a Gaussian filter in the horizontal direction and a derivative kernel in the vertical direction. Note that these two operations are equivalent to a Gaussian filter in a direction parallel to the curve, and a derivative filter normal to the curve. The resulting image is then binarized, as shown in Fig. 3, right. The columns in the NM space must be moved up and down in such a way that the central horizontal line intersects the white horizontal regions as much as possible. The idea is that $C_{k}$ must be attracted by the bright regions in the image, which correspond to the discontinuities in the tissue captured by the scanner. This procedure allows important local deformations of $C_{k}$ in the normal direction. For each control point $\left(x_{i}, y_{i}\right)$ of $C_{k}$ (that lies on the central horizontal line of the NM) we estimate the new "displacement" value $v_{d}^{i}$ as a weighted mean of each point in that vertical line, points that satisfy $\left(u_{i}, v\right)$ with $v \in[-d, d]$. The weights combine the area $A(u, v)$ of the connected component in which $(u, v)$ lies; $h(u, v)$ is the length of the maximal horizontal segment in that connected component and $|v|$, as a regularization term. The expression for the displacement $v_{d}^{i}$ is

$$
v_{d}^{i}=\lambda F_{s c}\left(u_{i}\right) \frac{\int_{-d}^{d} v|v| h\left(u_{i}, v\right) A\left(u_{i}, v\right) \mathrm{d} v}{\int_{-d}^{d}|v| h\left(u_{i}, v\right) A\left(u_{i}, v\right) \mathrm{d} v}
$$

where $\lambda$ is a global parameter estimated during the learning process. The local weight function $F_{s c}$, is either constant or the learned one as described in Section III-B. In the latter, $F_{s c}$ must 
be appropriately positioned on $C_{k}$. Due to the simplicity of the rib eye's contour, this can be done by choosing the starting point of the parametrization of $C_{k}$ in the same position as in the curves used to estimate $F_{s c}$.

\section{Shape Correction SteP}

The rib-eye shape information must be codified in such a way that encodes the experts' knowledge and is useful to the segmentation algorithm. In this context, the rib-eye shape is defined as the geometric property of this closed simple curve that is invariant to translations, rotations and scale. Given a curve, we need to quantify its shape, so that similarities between geometric shapes of different curves can be measured. There exist many shape descriptors in the literature [8], [10], [20]. We use the framework proposed by Klassen et al. [11], in which shapes are considered points in a given manifold, that allows for statistical analysis directly on this manifold. ${ }^{1}$ This representation will be used to model the rib-eye shape. In the following, we review the principal ideas. More information on this approach can be found in [11] and references therein.

\section{A. Shape Space Formulation}

1) Definition of the Shape Space: We consider only simple closed planar curves. The shape of a curve is the geometrical property invariant to position, orientation, and scale. A curve is parameterized with respect to the arc length by the parametrization $\alpha: \mathbb{R} \rightarrow \mathbb{R}^{2}$. To achieve invariance with respect to scale, the curve is scaled in such a way that the perimeter is $2 \pi$. Representing the curve with a direction function $\theta: \mathbb{R} \rightarrow \mathbb{R}$ gives position invariance. The value of $\theta(s)$ is the angle between the tangent vector $\dot{\alpha}(s)$ to the curve in $s$ and the horizontal axis. Direction functions of simple closed curves with rotation index 1 can be decomposed in the following way:

$$
\theta(s)=\theta_{0}(s)+f(s) \quad \text { for all } \quad s \in \mathbb{R}
$$

where $\theta_{0}(s)=s$ (a direction function of the unit circle) and $f$ is a periodic function with period $2 \pi$, that is square integrable in one period. Let $\mathbb{L}_{2}$ be the space of $2 \pi$ periodic square integrable functions, with the usual inner product. The space $\theta_{0}+\mathbb{L}_{2}$ is the set of functions that can be written as in (3). Our set of shapes is a subset of $\theta_{0}+\mathbb{L}_{2}$.

Given a curve with direction function $\theta$ we can define its mean value as $\bar{\theta}=(1 / 2 \pi) \int_{0}^{2 \pi} \theta(s) \mathrm{d} s$. We impose $\bar{\theta}=\pi$ to have rotation invariance. Let $\mathcal{C}$ be the set of direction functions with mean value $\pi$ and produced by closed curves of perimeter $2 \pi$

$$
\mathcal{C}=\left\{\theta \in \theta_{0}+\mathbb{L}^{2} \mid \frac{1}{2 \pi} \int_{0}^{2 \pi} \theta(s) \mathrm{d} s=\pi, \int_{0}^{2 \pi} e^{i \theta(s)} \mathrm{d} s=0\right\} .
$$

Even if invariant to translations, rotations and scales, the elements in $\mathcal{C}$ are not yet shapes. This is due to the different possible parametrizations of a curve. Let us consider the operator $\cdot[0,2 \pi] \times \mathcal{C} \rightarrow \mathcal{C}$ defined by $v \cdot \theta(s)=\theta(s-v)-v$. Although $\theta$

\footnotetext{
${ }^{1}$ There is some discussion about the precise characterization of this shape space formulation [24]. In this paper, we use this formulation and show that for our dataset, this framework gives good results.
}

and $v \cdot \theta$ are different points in $\mathcal{C}$ (the preshape space), they correspond to the same shape. Since the orbits $v \cdot \theta$ with $v \in[0,2 \pi]$ represent all arc length parametrizations of $\theta$, we can define an equivalence relation in $\mathcal{C}$

$$
\theta_{2} \sim \theta_{1} \Leftrightarrow \text { exists } v \in[0,2 \pi] \quad \text { such that } \theta_{2}(s)=v \cdot \theta_{1}(s) .
$$

The set of the classes of equivalence of $\mathcal{C}$ is the shape space $\mathcal{S}$. According to [11], $\mathcal{C}$ is a complete submanifold of co-dimension three.

2) Geodesic Paths: Both the preshape space $\mathcal{C}$ and the shape space $\mathcal{S}$ have a geometrical structure that allows geodesic paths to be defined between two points [11]. The distance between two such elements is then defined as the length of the geodesic path between them. The problem of finding geodesics in $\mathcal{S}$ reduces to the problem of finding geodesics in $\mathcal{C}$ that are orthogonal to the equivalence classes. These geodesics in $\mathcal{C}$ project onto geodesics in $\mathcal{S}$. This problem can be solved using a shooting method [11]. Let $\theta_{a}$ be an element of $\mathcal{C}$ and $f \in \mathcal{T}_{a}$ a direction vector, where $\mathcal{T}_{a} \subset \mathbb{L}^{2}$ is the tangent space of $\mathcal{C}$ at $\theta_{a}$. We will denote the geodesic flux from $\theta_{a}$ in the direction $f$ a certain time $t \in \mathbb{R}$ by $\Psi\left(\theta_{a}, t, f\right)$.

3) Discrete Shape Representation: In practice, we have discrete representations of the curves. A curve is then represented by $T$ ordered points of $\mathbb{R}^{2}$ with coordinates $\left(x_{i}, y_{i}\right)$. Let $C=\left[\left(x_{1}, y_{1}\right),\left(x_{2}, y_{2}\right), \ldots\left(x_{T}, y_{T}\right)\right]$ be a sampled curve. The spacing between samples is set to $\delta=\left\|\left(x_{i+1}, y_{i+1}\right)-\left(x_{i}, y_{i}\right)\right\|=2 \pi / T$, where $T$ is the number of samples. In practice, the used curves do not usually satisfy this condition. Therefore, a preprocessing step is required. We denote by $\phi_{n}$ the estimated direction function, computed as $\phi_{i}=\arctan \left(\left(y_{i+1}-y_{i}\right) /\left(x_{i+1}-x_{i}\right)\right)$ where $\left(x_{i}, y_{i}\right)$ are the coordinates of the sampled points. Next, the mean value $\bar{\phi}_{n}$ must be set equal to $\pi$ by adding a constant value, obtaining a single representation of this curve

$$
\theta_{i}=\phi_{i}-\bar{\phi}_{n}+\pi
$$

\section{B. Shape Statistics}

Following the experts' approach, we want to include the knowledge of the typical rib-eye shape and its more probable variations in our segmentation algorithm. This is done by studying the shape statistics of the database of marked US images, and then finding a parametric model that fits the data.

Two experts manually segmented 610 US images of animals from ten farms. This gives us a set of more than 1200 curves. The shape characteristics of the rib-eye are learned from 168 of the experts' traced curves. The marks are all simple closed curves. The framework proposed by Mio et al. [15] is used to analyze the data and fit a normal model to it. We assume the set of curves (or points $\theta_{i}$ in $\mathcal{S}$ ) has a normal multivariate probability density function (PDF) centered around a mean shape $\mu$ and with energy proportional to $d^{2}(\theta, \mu) / 2$.

The mean shape $\mu$ is computed as the Karcher mean, intrinsic to the manifold. It is estimated as the point $\mu$ in $\mathcal{S}$ that locally minimizes the function $V: \mathcal{S} \rightarrow \mathbb{R}$, given by $V(\theta)=\sum_{i=1}^{n} d\left(\theta, \theta_{i}\right)^{2}$, where $d\left(\theta, \theta_{i}\right)$ is the length of the geodesic path between $\theta$ and $\theta_{i}$. This intrinsic mean always 
exists, and is unique if the points $\left\{\theta_{1}, \ldots, \theta_{n}\right\}$ are sufficiently close [11]. Under this hypothesis, we find $\mu$ with a gradient descent algorithm.

The shape space in which we work is infinite dimensional and nonlinear. The nonlinearity is avoided by working in $\mathcal{T}_{\mu}$, the tangent space to the manifold at $\mu$. The idea is to fit the normal PDF in $\mathcal{T}_{\mu}$. The shape representatives in $\mathcal{T}_{\mu}$ are the vectors $f_{i}$ such that the geodesic from $\mu$ in the direction $f_{i}$ reaches $\theta_{i}$ in unit time. So we have that $\Psi\left(\mu, f_{i}, 1\right)=\theta_{i}$ for $i=1, \ldots, n$. The tangent space $\mathcal{T}_{\mu}$ is still infinite dimensional. To overcome this problem, we approximate $\mathcal{T}_{\mu}$ by the finite-dimensional subspace spanned by $\left\{f_{1}, \ldots, f_{n}\right\}$. Let $m \leqslant n$ be its dimension, and $\left\{e_{1}, \ldots, e_{m}\right\}$ an orthonormal basis of it. If the shapes under consideration are close enough, this approximation is valid.

Let $x_{i} \in \mathbb{R}^{m}$ be the coordinates of the vector $f_{i}$. In the coordinate space we estimate the parameters of a multivariate normal model with mean 0 and covariance matrix $K$. The eigenvectors and eigenvalues of $K$ give us the principal directions of the variation of the shapes and the variance in each direction. The PDF can be written as

$$
p(x)=\frac{1}{(2 \pi)^{m / 2} \operatorname{det}(K)^{1 / 2}} e^{-\frac{x^{\mathrm{T}} K^{-1} x}{2}} .
$$

Finally, the framework presented in [15] defines an associated energy function. Given a shape $\theta$, let $g_{\theta}$ be a vector in $\mathcal{T}_{\mu}$ that satisfies $\Psi\left(\mu, 1, g_{\theta}\right)=\theta$. The energy of the shape is calculated as

$$
E(\theta)=\frac{x_{\theta}^{\mathrm{T}} K^{-1} x_{\theta}}{2}+\frac{1}{\epsilon^{2}}\left\|g_{\theta}^{\perp}\right\|
$$

where $x_{\theta}$ are the coordinates of the projection of $g_{\theta}$ on the basis $\left\{e_{1}, \ldots, e_{m}\right\}, g_{\theta}^{\perp}$ is the component of $g_{\theta}$ in the normal space in $\mu$, and $\epsilon$ is a positive real number. The energy increases as the probability of the shape decreases. Note that both $K$ and $K^{-1}$ are positive definite matrices, so $E(\theta)$ is strictly positive for every $x_{\theta}$ not equal to zero. The vector $x_{\theta}=0$ has zero energy and corresponds to the mean shape $\mu$. Given that $V$ is an approximation of the tangent space, it is possible that $g_{\theta}$ lies outside of $V$. The second term in the energy penalizes that situation.

\section{Rib-Eye Shape}

Before fitting the Gaussian model to the set of manually detected curves, we must be sure that the data fulfills the hypotheses under which the model is valid. In particular, we want to study if there is any correlation between the shape of the rib-eye and the farms. We use a portion of the expert marked database to answer this question.

It is necessary to make some decisions about what curves should be considered before continuing this analysis. Usually, the rib-eye presents a fat intrusion, called hook, which appears in the upper left region of the image. This can be seen in Fig. 1. It represents, on average, less than $2 \%$ of the rib-eye area, and sometimes it is absent. Therefore, some experts do not take it into account. Given the small influence of the hook in terms of area, we decided to analyze its influence on the shape. We compared models that fit several sets of shapes with and without hook. The curve without hook is obtained by considering the convex hull for this section of the curve.

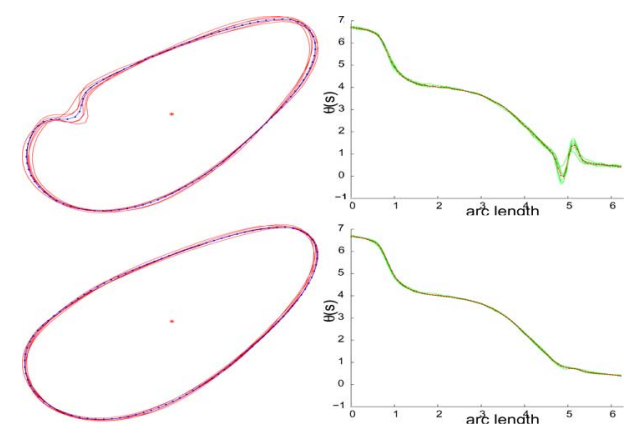

Fig. 5. Comparison of six farm mean shapes. Above, mean shapes computed considering the hook and the corresponding direction functions $\theta$ versus arclength. Below, mean shapes and their direction functions computed without hook.

We found that most of the shape variation is concentrated at the hook. Fig. 5 show a comparison between the mean shapes for data from six of the farms, both with and without hook. The mean shapes show different hooks, as one can tell from the top image in Fig. 5. In order to compare the covariance matrices of each cluster, we compared their eigendecomposition, the model has more variance when considering the hook. This suggests that it is a good strategy to work in a sort of multiresolution approach, as is done by the experts. First, try to fit a global rib-eye shape without the hook. Next, if the hook is present, try to find it. This last part is work in progress and will be reported elsewhere.

To see the effect of the principal variation modes we calculate the geodesic flux for a time proportional to the square root of the eigenvalue in the direction of the corresponding eigenvector. The most important variation is associated with the width of the rib-eye. The others, less important, are oscillatory perturbations around the mean shape. This experiment seems to show that there are not significative differences among farms. Using the convex hull in the hook region allows the entire dataset to be fit by a general simple Gaussian model.

\section{Shape Correction}

As mentioned earlier, a subset of 168 curves without hook was used to train the algorithm. As a result, we have the parameters of the normal PDF: the mean shape $\mu$ and the covariance matrix $K$. We develop two different ways to integrate this a priori knowledge into the segmentation algorithm. The main idea is to correct the control points of $C_{k}$, at iteration $k$, so that the shape of $C_{k+1}$ is closer to $\mu$.

1) Using the Mean Shape: $C_{k}$ is represented by its $n\left(x_{i}, y_{i}\right)$ control points

$$
C_{k}=\left[\left(x_{0}, y_{0}\right),\left(x_{1}, y_{1}\right), \ldots,\left(x_{n-1}, y_{n-1}\right)\right] .
$$

The shape correction step is as follows.

a) Find the shape space representation, $\theta_{k}$, of $C_{k}$.

b) Estimate the geodesic path between $\theta_{k}$ and the mean shape $\mu$, represented by $f_{k}$, where $\Psi\left(\theta_{k}, 1, f_{k}\right)=\mu$.

c) Move along the geodesic path from $\theta_{k}$ towards $\mu$, by: $\theta_{k+1}=\Psi\left(\theta_{k}, t_{k}, f_{k}\right)$ with $t_{k} \in[0,1]$.

d) Go back to the image space, i.e. find $C_{k+1}$. Knowing $\theta_{k+1}$, we must fix the correct scale, orientation and position for the new curve. This is done by registering the 

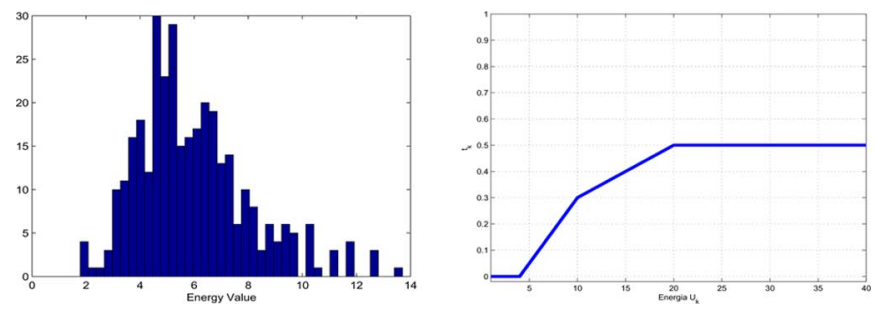

Fig. 6. Left: Histogram of the energy for 100 expert traced curves. Right: Mapping function $F_{\text {map }}$ as a function of the curve energy, used to weight the amount of progress along the geodesic path in the shape correction step.

image shape defined by $\theta_{k+1}$ with the control points of $C_{k}$. For this, we use the approach presented in [13].

2) Using the Statistics: The basic idea is to use the energy defined in (7) to decide the amount of progress along the geodesic path from $\theta_{k}$ towards $\mu$. We include here again the $a$ priori knowledge. The idea is to progress more for curves with low probability of being a rib-eye

$$
\theta_{k+1}=\Psi\left(\theta_{k}, t_{k}\left(E\left(\theta_{k}\right)\right), f_{k}\right) \quad \text { with } t_{k} \in[0,1]
$$

where $t_{k}\left(E\left(\theta_{k}\right)\right)=F_{\text {map }}\left(E\left(\theta_{k}\right)\right), F_{\text {map }}: \mathbb{R}^{+} \rightarrow[0,1]$. Note that we control the weight of the shape correction step modulating the amount of time in the geodesic flux $\Psi\left(\theta_{k}, t, f_{k}\right)$. Fig. 6 shows the energy distribution for 100 expert marked curves. The idea is that for energy values close to most of the experts' marks, the shape correction step must play a less important role. The weight of the shape correction factor must increase as the energy of the curve under consideration is far from the experts' mean energy. A threshold guarantees a shape correction factor never greater than half of the total geodesic path to $\mu . F_{\text {map }}$ is a piecewise linear function (see Fig. 6).

\section{INITIAL CONDITION}

To avoid local minima and obtain quick convergence, it is important to have a good initialization. The main idea is to fit the mean shape $\mu$ in the image to be segmented, and use that curve as the starting point. We must find its right position, orientation and scale. The initial condition algorithm is as follows. For technical details, refer to [1].

a) Preprocess the image. A region of interest (ROI) window is applied to eliminate the scanner artifacts such as the date, scale, animal ID (see Fig. 3). The ROI is defined manually once for all the images taken with the same equipment (scanner and probe). Then the following operations are applied to the ROI:

- anisotropic diffusion in order to reduce speckle noise;

- high-pass filtering, with a very low cutoff frequency, is applied to compensate for the slow bright variations in the image;

- binarization using a global threshold proportional to the gray level variance in the ROI;

- elimination of small regions and labeling.

The parameters for the steps described above are fixed for all the US images acquired with the same equipment. Let $I_{p}$ be this preprocessed image (an example is in Fig. 7). The big regions of the binarized image roughly delimit the rib-eye.

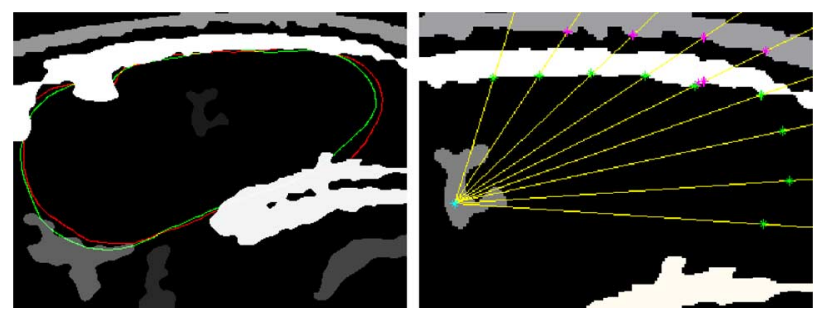

Fig. 7. Left: Preprocessed image, expert traced curve (green and red). Right: Ppoints of the rib-eye contour determined by the intersection between the rays and the regions in the image $I_{p}$.

b) Fix a seed point $P_{s}$ inside the large regions, approximately equidistant to all of them, to define the initial position of the curve.

c) Determine $n$ points of the rib-eye contour. This is done drawing rays at equally spaced angles originating from $P_{s}$ (see Fig. 7). The curve is defined by the intersections between these rays and the regions in $I_{p}$. The aim is for each ray to find one point of the rib-eye's contour. As seen in Fig. 7, for some rays, this is difficult. This can be due to the absence of intersection or to multiple intersections between the ray and the binarized regions. In both cases we use the information of neighbor rays in order to maintain the curve continuity.

d) The marked points determine a noisy curve for which the position is roughly close to the rib-eye borders. We correct this initial estimation by fitting the mean shape curve to this set of points as in [13].

\section{EXPERIMENTAL RESULTS}

\section{A. Measurements}

To analyze the performance of the algorithm we must define similarity measures between two (closed) curves: the ground truth $C_{T}$ (in this case, the expert traced curve) and the curve $C$ produced by the segmentation algorithm. Let us denote by $R_{C} \subset \mathbb{R}^{2}$ the inner region of $C$. Let $A\left(R_{C}\right)$ be the area of $R_{C}$. We define the following measures.

- Intersection to union area ratio:

$$
M_{R}=1-\left(A\left(R_{C_{T}} \cap R_{C}\right) / A\left(R_{C_{T}} \cup R_{C}\right)\right) .
$$

- Area difference. Although the goal of the algorithm is to segment the rib-eye by finding a curve as similar as possible to the expert's traced curve, the important measurement in terms of meat quality is the rib-eye area. The area error is measured as: $M_{A}=\left(\left|A\left(R_{C_{T}}\right)-A\left(R_{C}\right)\right| / A\left(R_{C_{T}}\right)\right)$.

\section{B. Comparison Between Experts}

Analyzing the experts' manual segmentations gives us an idea of the difficulty of the problem, as well as the level of performance we should expect from the algorithm. Table I, shows the measurements $M_{R}$ and $M_{A}$ for the images marked by both experts. Note that for the (shape oriented) indicator $M_{R}$, the experts agree in $60 \%$ of the cases to a $10 \%$ error range. Concerning the area indicator $M_{A}$, the experts agree to $5 \%$ error in $52 \%$ of 
TABLE I

COMParison Between EXPerts' Detections. Percentage of IMAges FOR WHICH THE MEASUREMENTS ARE IN THE ERROR RANGES

\begin{tabular}{|l||c|c|c|c|c|}
\hline Error range (\%) & $(0-5)$ & $(0-10)$ & $(0-15)$ & $(0-20)$ & $(0-25)$ \\
\hline \hline$M_{R}$ & 2.46 & 63.12 & 93.61 & 98.69 & 99.67 \\
\hline$M_{A}$ & 52.79 & 87.38 & 97.54 & 99.34 & 99.83 \\
\hline
\end{tabular}
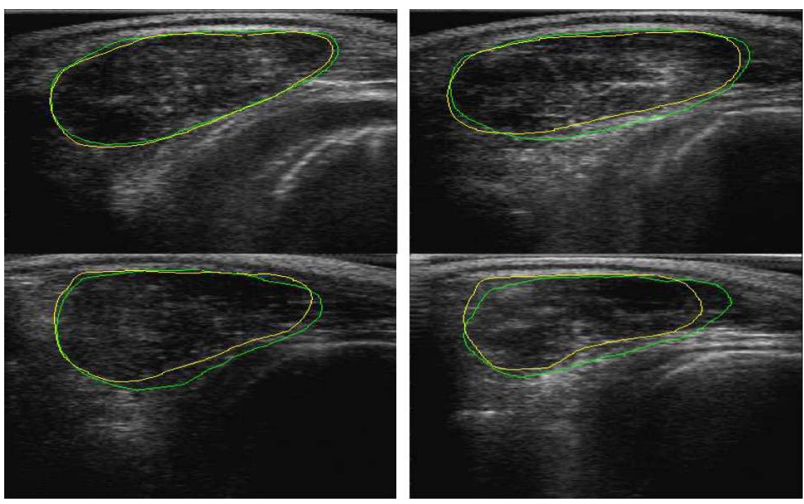

Fig. 8. Examples of experts' marks. From top left to bottom right, the $M_{R}$ values are $0.068,0.14,0.15$, and 0.23 .

TABLE II

Automatic Algorithm Versus Experts. Percentage of IMAges FOR WHICH THE MEASUREMENTS ARE IN THE ERROR RANGES

\begin{tabular}{|c|c||c|c|c|c|}
\hline & Expert $\backslash$ Error range (\%) & $(0-5)$ & $(0-10)$ & $(0-15)$ & $(0-20)$ \\
\hline \hline$M_{R}$ & Expert 1 & 0.86 & 30.24 & 69.42 & 88.49 \\
\hline$M_{R}$ & Expert 2 & 0.34 & 25.38 & 61.67 & 84.16 \\
\hline$M_{R}$ & Closest expert & 1.20 & 40.48 & 75.30 & 90.74 \\
\hline \hline$M_{A}$ & Expert 1 & 38.83 & 67.18 & 81.62 & 91.24 \\
\hline$M_{A}$ & Expert 2 & 36.47 & 59.97 & 76.15 & 87.56 \\
\hline$M_{A}$ & Closest expert & 44.94 & 71.18 & 84.05 & 93.14 \\
\hline
\end{tabular}

the marks. These results are in agreement with inter expert variability measurements reported elsewhere [9]. Fig. 8 illustrates the first four $M_{R}$ ranges of Table I. More than $98 \%$ of the experts' marks are inside these ranges. Therefore, we consider a good detection if $M_{R} \leq 0.2$.

\section{Results}

We compare the performance of the proposed automatic algorithm with the experts' manual segmentation. Table II summarizes the results over the set of 610 images. The algorithm parameters were fixed once, and no tuning was made for each image.

The SW is written in $\mathrm{C}++$. Tests were performed on a 2.8-GHz Xeon under WindowsXP. The mean time for the segmentation of an US image is $5.1 \mathrm{~s}$. Convergence is reached on average in 24 iterations. Only in 15\% of the cases the algorithm reaches the maximum number of iterations. Image dimensions are $360 \times 400$ pixels. In both cases the more rigorous measure
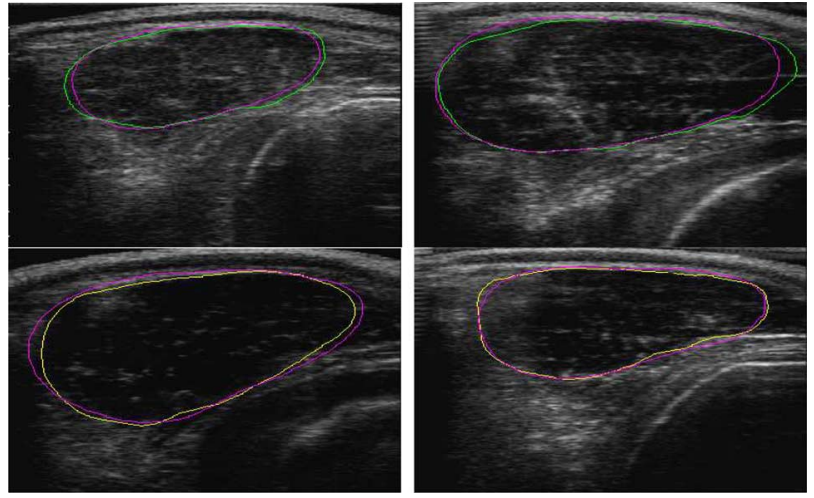

Fig. 9. Some results. Green curves for Expert 1, yellow curves for Expert 2, and magenta curves for automatic algorithm.

$M_{R}$ performs well for more than $84 \%$ of the images. Comparing the algorithm results with the expert's segmentation closer to the automatic solution, we obtain a $M_{R} \leq 0.2$ for $90 \%$ of the images. This means that in $90 \%$ of the cases, the detection agrees with at least one expert. The performance for the measurement $M_{A}$ is naturally better as can be seen in the first ranges.

The initial condition plays an important but not a critical role. Using a fixed initialization (the mean shape centered in the ROI), the $M_{R}$ against the Expert 1 drops from $88 \%$ to $82 \%$.

We consider the results for which $M_{R} \geq 0.5$, to be outliers. The percentage of outliers is $1.5 \%$ for Expert 1 and $1 \%$ for Expert 2 . The outliers are generated by several reasons. Very poor image quality, sometimes the curve is caught by a local minimum due to bad initialization, or the algorithm is attracted by other structures present in the US image. Improvements to the algorithm that can handle these situations are the object of current work. Fig. 9 contains several examples of the automatic segmentation for some US images along with the experts' manual segmentation for comparison.

\section{CONCLUSION}

In this paper, we have presented a shape prior segmentation method applied to the automatic measure of rib-eye area in US images. The proposed method is an iterative two-step algorithm composed of an image and a shape steps. The algorithm deforms a curve using image information and a shape prior in such a way that the final curve fits the existing boundary structures of the rib-eye in the US image and has the shape of a likely rib-eye. The algorithm is not formulated in a variational way; however, it gives good results in real time. As part of our ongoing current work, we are studying a variational formulation to compare both approaches. The proposed method is general and can be applied to other applications, when the target shape is a 2-D simple closed curve. The algorithm's performance allows for real time measurement of the rib-eye area from US images. We present extensive experiments with real data. The automatic results are in good agreement with the expert marked curves on the same images. There are many possible improvements to the algorithm. We are currently studying other ways to model the shape prior knowledge. For example, in [14], the framework is extended to allow stretching of the shapes. This can facilitate the 
inclusion of the hook in the shape prior. Furthermore, the shape of the rib-eye can be learned in a nonparametric fashion, instead of modeling it by a Gaussian. We are also carrying on extensive validation over an expert marked image data base composed of several thousand of US images.

\section{ACKNOWLEDGMENT}

The authors would like to thank D. Gimeno, A. Pardo, O. Gil, G. Sapiro, and S. Levine for fruitful discussions; the reviewers for the critiques that help us improve the paper; as well as the experts A. Calistro and D. Correa for their important work and comments. The set of US images has been taken, marked, and provided by the Instituto Nacional de Investigación Agropecuaria, Uruguay.

\section{REFERENCES}

[1] P. Arias, A. Pini, G. Sanguinetti, and P. Sprechmann, "Segmentación con información a priori de forma aplicada a sistema de valoración cárnica," E.E. thesis, Univ. República, Montevideo, Uruguay, 2005.

[2] P. Cancela, F. Reyes, P. Rodríguez, G. Randall, and A. Fernández, "Automatic object detection using shape information in ultrasound images," in Proc. 3rd Int. Conf. Image Process., 2003, pp. 417-420.

[3] V. Caselles, R. Kimmel, and G. Sapiro, "Geodesic active contours," in Proc. Int. Conf. Computer Vision, 1995, pp. 694-699.

[4] T. Chan and W. Zhu, "Level set based shape prior segmentation," Tech. Rep. 03-66, Comput. Appl. Math., Univ. California, Los Angeles, 2003.

[5] G. Charpiat, O. Faugeras, and R. Keriven, "Approximations of shape metrics and application to shape warping and empirical shape statistics," Found. Comput. Math., vol. 5, no. 1, pp. 1-58, 2005.

[6] Y. Chen, H. Tagare, S. Thiruvenkadam, F. Huang, D. Wilson, K. Gopinath, R. Briggs, and E. Geiser, "Using prior shapes in geometric active contours in a variational framework," Int. J. Comput. Vis., vol. 50, no. 3, pp. 315-328, 2002.

[7] D. Cremers, N. Sochen, and C. Schnorr, "Towards recognition-based variational segmentation using shape priors and dynamic labeling," in Proc. Int. Conf. Scale Space Theory Computer Vision, 2003, vol. 2695, pp. 388-400.

[8] I. Dryden and K. Mardia, Statistical Shape Analysis. New York: Wiley, 1998.

[9] W. O. Herring, D. C. Miller, J. K. Bertrand, and L. L. Benyshek, "Evaluation of machine, technician, and interpreter effects on ultrasonic measures of backfat and longissimus muscle area in beef cattle," J. Animal Sci., vol. 72, no. 9, pp. 2216-2226, 1994.

[10] D. G. Kendall, D. Barden, T. K. Carne, and H. Le, Shape and Shape Theory. New York: Wiley, 1999.

[11] E. Klassen, A. Srivastava, W. Mio, and S. Joshi, "Analysis of planar shapes using geodesic paths on shape spaces," IEEE Trans. Pattern Anal. Mach. Intell., vol. 26, no. 3, pp. 372-383, Mar. 2003.

[12] M. Leventon, E. Grimson, and O. Faugeras, "Statistical shape influence in geodesic active controus," in Proc. Computer Vision Pattern Recognition Conf., 2000, pp. 316-322.

[13] J. S. Marques and A. J. Abrantes, "Shape alignment-Optimal initial point and pose estimation," Pattern Recognit. Lett., vol. 18, no. 1, pp. 49-53, 1997.

[14] W. Mio and A. Srivastava, "Elastic-string models for representation and analysis of planar shapes," presented at the Computer Vision Pattern Recognition Conf., 2004.

[15] W. Mio, A. Srivastava, and X. Liu, "Learning and bayesian shape extraction for object recognition," in Proc. 4th ECCV, 2004, pp. 62-73.

[16] U. S. Dept. Agriculture, 1997, U.S. Standards for Grades of Carcass Beef [Online]. Available: http://www.ams.usda.gov/standards/beef-car.pdf

[17] N. Paragios, "A variational approach for the segmentation of the left ventricle," Int. J. Comput. Vis., pp. 345-362, 2002.

[18] M. Rousson and N. Paragios, "Shape priors for level set representations," in Proc. 2nd ECCV, 2002, pp. 78-92.
[19] A. U. Services, 2005. Ithaca, NY [Online]. Available: http://people. clarityconnect.com/webpages/aus/AUSPRODUCTS.HTM

[20] C. G. Small, The Statistical Theory of Shape. New York: Springer, 1996.

[21] R. G. Tait, D. E. Wilson, and G. H. Rouse, "Prediction of retail product and trimmable fat yields from the four primal cuts in beef cattle using ultrasound or carcass data," J. Animal Sci., vol. 83, pp. 1353-1360, 2005.

[22] M. Technology, 2006 [Online]. Available: http://www.microbeef.com/

[23] A. Tsai, A. Yezzi, W. Wells, C. Tempany, D. Tucker, A. Fan, E. Grimson, and A. Willsky, "A shape-based approach to the segmentation of medical imagery using level sets," IEEE Trans. Med. Imag., vol. 22, no. 2, pp. 137-154, Feb. 2003.

[24] A. Yezzi and A. Mennucci, 2005, Metrics in the Space of Curves [Online]. Available: http://arxiv.org/abs/math/0412454

[25] H. L. Zhang, D. E. Wilson, and G. H. Rouse, "Image analysis and application systems in quality evalutaion and prediction for meat and live meat animals," in Proc. 3rd Int. Conf. Image Processing, 1994, pp. 241-244.

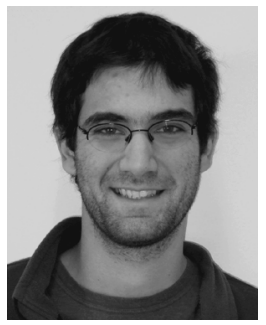

Pablo Arias received the E.E. degree in 2006 from the Universidad de la República, Montevideo, Uruguay, where he is currently pursuing the M.Sc. degree in electrical engineering.

He has a visiting position in the Electrical Engineering Department, University of Minnesota, Minneapolis.

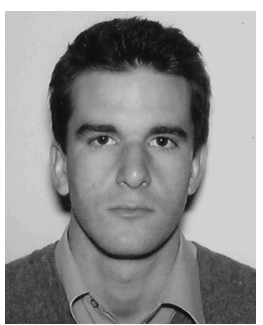

Alejandro Pini is an electrical engineering student at Universidad de la República, Montevideo, Uruguay.

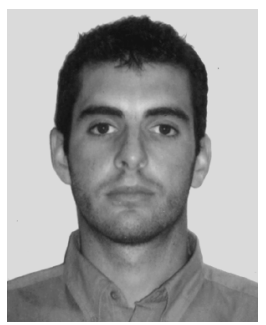

Gonzalo Sanguinetti received the E.E. degree in 2005 from the Universidad de la República, Montevideo, Uruguay, where he is currently pursuing the doctoral degree in electrical engineering.

$\mathrm{He}$ is also a visiting student at the Università di Bologna, Bologna, Italy.

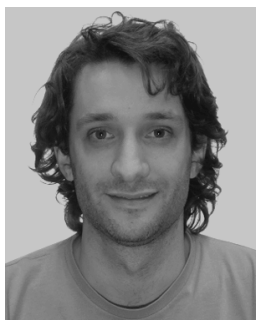

Pablo Sprechmann received the E.E. degree in 2006 from the Universidad de la República, Montevideo, Uruguay, where he is currently pursuing the M.Sc. degree in electrical engineering.

During the year 2006, he had a visiting position in the Electrical Engineering Department, University of Minnesota, Minneapolis. 


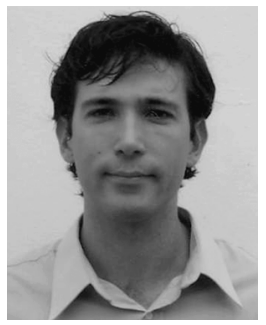

Pablo Cancela received the E.E. and C.S. degrees in 2002 from the Universidad de la República, Montevideo, Uruguay, where he is currently pursuing the M.Sc. degree in electrical engineering.

He was a visiting student at INRIA in 2003.

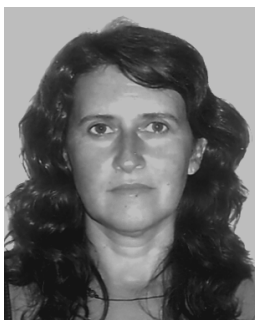

Alicia Fernández received the E.E. degree in 1990 from the Universidad de la República (UR), Montevideo, Uruguay, where she is currently an Associate Professor in the Electrical Engineering Department.

She was Head of the Telecommunications Department at UR from 2003 to 2005 and co-leads the Group of Image Processing at UR. Her research interests are in image processing and pattern recognition.

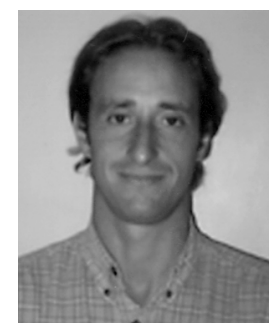

Alvaro Gómez received the E.E. degree in 1998 and the M.Sc. degree in electrical engineering in 2003 from Universidad de la República (UR), Montevideo, Uruguay.

He is currently an Adjoint Professor with the Electrical Engineering Department, UR.

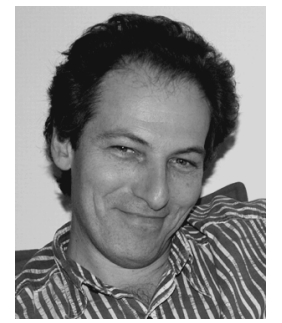

Gregory Randall received the E.E. degree in 1983 form ISPJAE, Cuba, the DEA in robotics from Paris VI, and the Ph.D. degree in applied informatics from Paris XI in 1991.

$\mathrm{He}$ is a Professor in the Electrical Engineering Department at the Universidad de la República (UR), Montevideo, Uruguay. From 1988 to 1991, he was a member of the Robotvis Project, INRIA. From 1992 to 1994 , he was with COSE, Paris. In 1994, he joined UR, where he founded the Image Processing Group. From 1998 to 2004, he was the Head of the Electrical Engineering Department, UR. During 2005-2006, he was a Visiting Professor in the Electrical and Computer Engineering Department and IMA general member, University of Minnesota, Minneapolis. His research interests are in image processing and computer vision. 